British Journal of Nutrition (2020), 124, 844-852

doi: $10.1017 / \mathrm{S} 0007114520001762$

(C) The Author(s) 2020. This is an Open Access article, distributed under the terms of the Creative Commons Attribution licence (http:// creativecommons.org/licenses/by/4.0/), which permits unrestricted re-use, distribution, and reproduction in any medium, provided the original work is properly cited.

\title{
Vegetarian, pescatarian and flexitarian diets: sociodemographic determinants and association with cardiovascular risk factors in a Swiss urban population
}

\author{
Hannah Wozniak ${ }^{1} \dagger$, Christophe Larpin ${ }^{1} \dagger$, Carlos de Mestral $^{2}$, Idris Guessous ${ }^{2}$, Jean-Luc Reny ${ }^{1}$ and \\ Silvia Stringhini ${ }^{2,3 * *}$ \\ ${ }^{1}$ Department of General Internal Medicine, Geneva University Hospitals, 1205 Geneva, Switzerland \\ ${ }^{2}$ Unit of Population Epidemiology, Department of Primary Care Medicine, Geneva University Hospitals, 1205 Geneva, \\ Switzerland \\ ${ }^{3}$ University Centre for General Medicine and Public Health, University of Lausanne, 1011 Lausanne, Switzerland
}

(Submitted 18 November 2019 - Final revision received 10 May 2020 - Accepted 12 May 2020 - First published online 18 May 2020 )

Abstract

Prevalence and trends of different vegetarian diets remain unknown, with estimates varying depending on the source. Evidence suggests that vegetarian diets are associated with a more favourable cardiovascular risk profile. The present study aimed to assess the prevalence and trends of different types of vegetarian diets in a population-based representative sample, sociodemographic characteristics of participants following such diets and the association of these diets with cardiovascular risk factors. Using repeated cross-sectional population-based surveys conducted in Geneva, Switzerland, 10797 individuals participated in the study between 2005 and 2017. Participants were classified as vegetarians, pescatarians, flexitarians or omnivores using an FFQ. Sociodemographic and cardiovascular risk factors were evaluated through questionnaires, anthropometric measurements and blood tests. Findings show prevalence of vegetarians increased from 0.5 to $1.2 \%$, pescatarians from 0.3 to $1.1 \%$ and flexitarians remained stable at $15.6 \%$ of the population over the study period. Compared with omnivores, vegetarians were more likely to be young (OR 2.38; $95 \%$ CI 1.01, 5.6), have higher education (OR 1.59; $95 \%$ CI 1.01, 2.49) and lower income (OR 1.83; $95 \%$ CI 1.04, 3.21); pescatarians and flexitarians were more likely to be women (pescatarian: OR 1.81; $95 \%$ CI 1·10, 3.00; vegetarian: OR 1.57; $95 \%$ CI $1.41,1.75)$ and flexitarians were also more likely to have a lower income (OR 1.31; $95 \%$ CI 1.13, 1.53). Participants who adhered to any diet excluding/reducing meat intake had lower BMI, total cholesterol and hypertension compared with omnivores. The present study shows an increase in the prevalence of vegetarians over a 13-year period and suggests that the different vegetarian diets assessed are associated with a better cardiovascular risk profile.

Key words: Vegetarian diets: Pescatarian diet: Flexitarian diet: Vegetarian prevalence: Cardiovascular risk factors: Sociodemographic factors: Diet trends

In Western countries, it is increasingly popular to consciously reduce the consumption of meat, mainly due to environmental, ethical and health concerns ${ }^{(1,2)}$. However, the proportion of the population following vegetarian or flexitarian diets remains unknown as estimates vary widely depending on the source ${ }^{(2)}$. When estimates are driven by online or telephone surveys sponsored by vegetarian associations, the prevalence of self-reported vegetarians can be up to $10-14 \%^{(2-5)}$. However, in populationbased studies, national surveys, or independent analysis, only $1-4 \%$ of the population reports following a vegetarian $\operatorname{diet}^{(6-14)}$.

An accurate estimation of the prevalence of individuals following a vegetarian diet is also complicated by the fact that a large number of different types of dietary regimens fall under the definition of 'vegetarian'. Individuals may define themselves as vegetarians depending on the questions that are being asked or on the definition adopted in the specific survey. Indeed, different types of vegetarian diets can be identified. The strictest regimen is the vegan diet, where all animal products are avoided. Lacto-ovo vegetarians, simplified as vegetarians, include in their diet animal-derived products such as eggs and dairy products, but exclude meat or fish. Pescatarians are defined as vegetarians who also consume fish and seafood products ${ }^{(1,15)}$. More recently, the flexitarian diet (or semi-vegetarian) has been gaining popularity and identifies individuals eating meat occasionally, with no clear consensus on quantities of meat consumed per week or month required for falling under this category ${ }^{(16)}$. Omnivores are defined as eating indifferently animal and plant products.

In the current literature, it is difficult to find reliable data on the prevalence of vegetarians in the general population, and there is even less data on trends in the prevalence of these diets.

* Corresponding author: Dr Silvia Stringhini, email silvia.stringhini@hcuge.ch

$\dagger$ These authors contributed equally to this work. 
Furthermore, few studies have attempted to characterise individuals following a vegetarian diet. Most of these reported that individuals following a vegetarian diet tend to be women, younger, smoke less and have a higher education ${ }^{(9,17)}$. Nonetheless, there seems to be contradictory evidence about the link between income and following a vegetarian $\operatorname{diet}^{(9,18)}$.

The medical community is showing growing interest in the potentially beneficial effects of a vegetarian diet, as shown by the increasing number of studies on the subject ${ }^{(19-23)}$. Cardiovascular risk factors such as elevated BMI, hypertension, hypercholesterolaemia and diabetes seem to be less prevalent in the population following different vegetarian diets ${ }^{(22,24-26)}$. Furthermore, some meta-analyses have shown a link between vegetarian diet and a protective effect on IHD and certain cancers $^{(21)}$. Finally, some but not all evidence suggests a link between following a vegetarian diet and reduced all-cause mortality $(27,28)$

The main purpose of the present study is to reliably assess the prevalence and temporal trends of different types of vegetarian diets in a yearly Swiss cross-sectional study. The secondary objectives are to analyse the sociodemographic characteristics of the cohort and to assess the possible correlation between the different types of vegetarian diets and cardiovascular risk factors. With the presumed health benefits of different vegetarian diets, it is essential to better identify and characterise who in the general population is following such diets.

\section{Materials and methods}

Data were collected using the Bus Santé study, a cross-sectional population-based study in the State of Geneva, Switzerland conducted annually since $1993^{(29,30)}$. The aim is to collect sociodemographic data and information about cardiovascular risk factors from a representative sample of the Swiss adult population. Each year, about 1000 participants aged from 18 to 74 years are selected to participate in the study and individuals cannot be included more than once in the study. Eligible participants are identified from an annual residential list made by the Government of Geneva. Potential participants are contacted first by mail. If no answer is given, eligible participants can receive up to seven phone calls and up to two more mails. People who do not answer are replaced by another eligible participant. People who refuse to participate are not replaced. Participants are met either at the Geneva University Hospital or at a mobile medical unit (a bus), facilitating access to all suburban areas. Participation rate varied between 45 and $55 \%$ with yearly variations.

First, participants receive a questionnaire at home about lifestyle habits (smoking, physical activity and nutrition), sociodemographic information and health, including questions regarding cardiovascular risks factors. The dietary habits are assessed using an FFQ with a recall period of 4 weeks. Secondly, participants undergo a face-to-face interview with trained nurses who verify the completeness of the questionnaires and take anthropometric measures including height and weight, according to standard procedures. For weight measurement, the participant is weighed on a medical scale and has to take off his shoes and be lightly dressed. Height is measured using a medical gauge. Blood pressure is measured three times after $10 \mathrm{~min}$ of rest in a sitting position with a cuff size adjusted to the arm circumference. The average of the three blood pressure measures is used for the analyses. A blood test is performed including the measurement of fasting plasma glucose, total cholesterol and TAG.

The Bus Santé study complied with the declaration of Helsinki and is approved by the institutional ethical committee of the University of Geneva. Furthermore, patients gave their written consent. No financial compensation was given to participants.

\section{Food frequency questionnaire}

The FFQ used in the present study has been validated in the Geneva population $^{(31)}$ and has been used in the previous studies $^{(32,33)}$. It is based on ninety-seven different food items.

For each item, the participant must indicate the size of the portion consumed (smaller, equal or larger than a reference size) and the frequency at which it was consumed over the last 4 weeks $(<1 \times \text { over the last } 4 \text { weeks up to twice a day })^{(34)}$.

\section{Type of diet}

Participants were classified according to the type of diet based on the FFQ results. Participants were classified as omnivores when they ate meat $>1$ /week; as vegetarians when they excluded red meat, poultry and fish from their diet but ate dairy products and eggs; as pescatarians when they consumed fish, in addition to dairy products and eggs but did not eat red meat or poultry ${ }^{(15)}$ and as flexitarians when they included eggs and dairy products in their daily diet and red meat or poultry at a frequency of $\geq 1 /$ month but $\leq 1 /$ week $^{(19,28,35)}$.

\section{Cardiovascular risk factors}

We chose to focus on five cardiovascular risk factors: overweight, hypertension hypercholesterolaemia, diabetes and smoking.

BMI was calculated as weight/height ${ }^{2}$. Participants were classified as having normal BMI (BMI $\left.<25 \mathrm{~kg} / \mathrm{m}^{2}\right)$, as being overweight (BMI $\geq 25$ and $<30 \mathrm{~kg} / \mathrm{m}^{2}$ ) or as being obese $\left(\mathrm{BMI} \geq 30 \mathrm{~kg} / \mathrm{m}^{2}\right.$ ). Hypertension was defined as either one measurement of the systolic blood pressure $\geq 140 \mathrm{mmHg}$ or mean blood pressure $\geq 90 \mathrm{mmHg}$ or if medications against high blood pressure were taken or as having a previous diagnosis of hypertension. Hypercholesterolaemia was defined as having total blood cholesterol $>6.5 \mathrm{mmol} / 1$ and $\mathrm{HDL}<1 \mathrm{mmol} / \mathrm{l}$ or if medications against hypercholesterolaemia were taken or as having a previous diagnosis. Diabetes was defined as a fasting glucose $>7 \mathrm{mmol} / \mathrm{l}$ or if medications against diabetes were taken or as having a previous diagnosis. Smoking was defined as being a current smoker.

Total cholesterol, systolic and diastolic blood pressure, fasting plasma glucose and BMI were also assessed as continuous biomarkers in relation to the different types of diet. 
Table 1. Description of sample, Bus Santé study 2005-2017 (n 10 797)*

(Numbers and percentages; mean values and standard deviations)

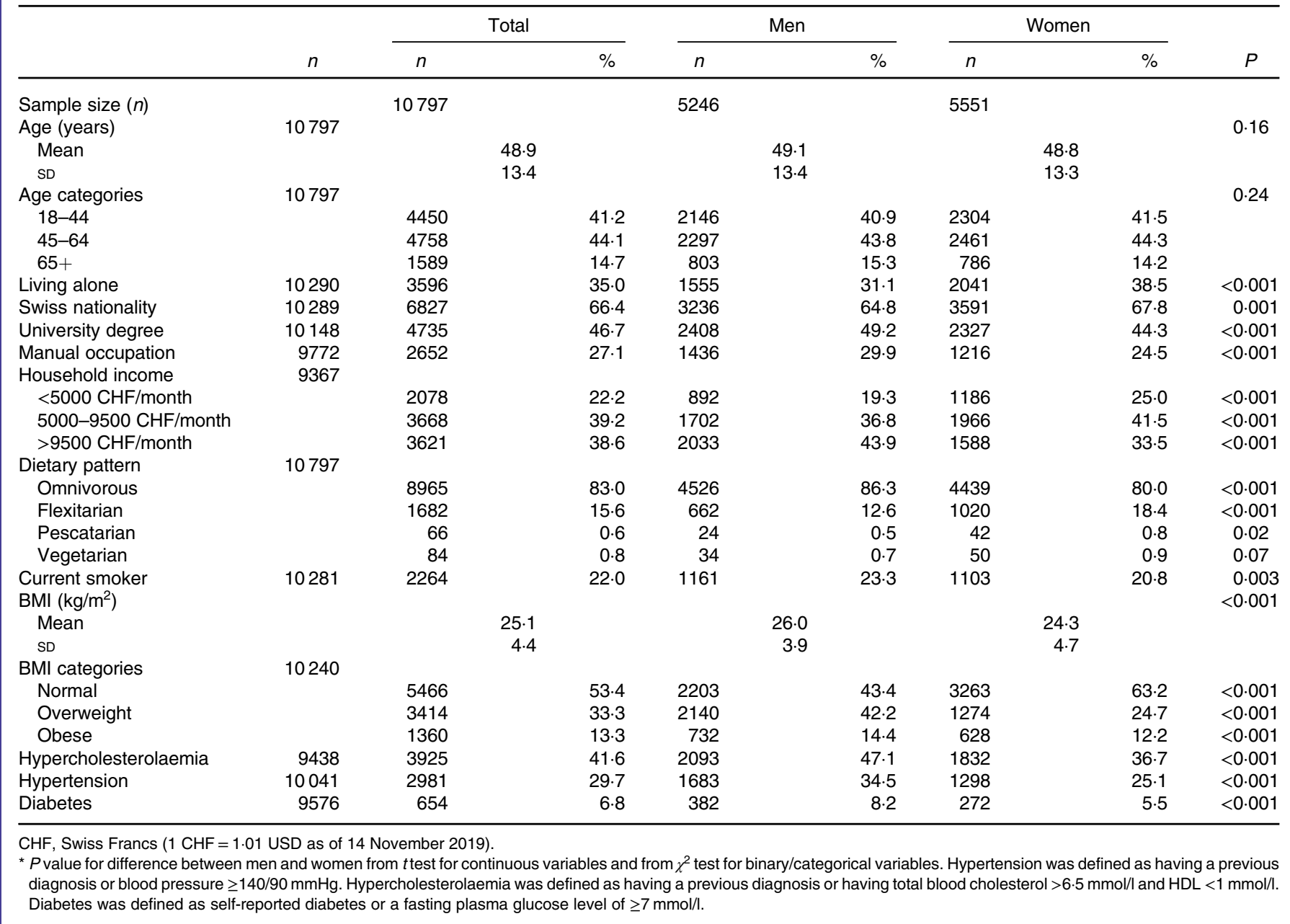

\section{Statistical analysis}

The analytical sample comprised all the participants included in the Bus Santé study from 2005 to 2017. Although the Bus Santé study started in 1993, biomarkers of interest for our study became available from 2005 onwards, which is why earlier data were not included in the analysis. We used $\chi^{2}$ test for differences in dietary pattern between men and women in binary/categorical variables and student $t$ test in continuous outcomes. All assumptions were met for normality and homoscedasticity. Given the sex differences in diet patterns, we conducted analyses separately for men and women, as well as in the overall sample. To calculate the prevalence and $95 \% \mathrm{CI}$, we applied margins after logistic regression, adjusting for age in sex-specific models and for age and sex in the overall model. To assess the change in prevalence between the first $\left(\%_{\text {start }}\right)$ and last $\left(\%_{\text {last }}\right)$ survey periods, we used the following formula: $\left(\left(\%_{\text {last }}-\%_{\text {start }}\right) /\left(\%_{\text {start }}\right)\right) \times 100$. To test for linear trend in the prevalence of diet patterns, we included the survey period variable as continuous predictor in the model. To assess for trends in food groups (i.e. meat type consumption) and for the association between diet patterns and biomarkers, we applied multivariable linear regression. To investigate the association between diet patterns and sociodemographic and cardiovascular risk factors, we used multinomial logistic regression, adjusting the estimates for age, sex and survey period. We further adjusted the estimates for BMI in sensitivity analyses to assess its role in the link between diet type and cardiovascular risk factors. All statistical analyses were conducted using STATA version 15 (Stata Corp.). The level of significance was set to $P<0.05$. Results for daily dietary intake are expressed as $\beta$-coefficients and $95 \% \mathrm{CI}$, for biomarkers as means and standard deviations and for the association between diet patterns and sociodemographic and cardiovascular risk factors as OR and $95 \% \mathrm{CI}$.

\section{Results}

\section{Baseline characteristics and prevalence}

A total of 10797 participants were included in the analyses, which spanned over a period of 13 years, from 2005 until 2017 (Table 1). Of the total number of participants, $51 \%$ were women and the mean age was 48.9 (SD 13.4) years. The mean BMI was 25.1 (sD 4.4 ) $\mathrm{kg} / \mathrm{m}^{2}$, with $33.3 \%$ of participants being overweight and $13.3 \%$ being obese. Current smokers were 
$22 \%$ of the sample. In the sample, $46.7 \%$ had been through higher education or had a university degree.

Within the studied population, there were eighty-four vegetarians (0.8\%), sixty-six pescatarians (0.6\%) and 1682 flexitarians $(15.6 \%)$. Due to the extremely low prevalence of vegans ( $<10$ people over the 13 years of data collection), no sub-group analysis was conducted for this category.

\section{Trends for diets and meat consumption}

Over the 13 year study period, the prevalence of a vegetarian diet increased from 0.5 to $1.2 \%(P=0.03$; Table 2$)$. In analysis stratified by sex, an increase in the prevalence of vegetarians was significant for women but not for men. The proportion of participants following a pescatarian diet also increased significantly from 0.3 to $1.1 \%(P<0.01)$ with a significant increase for men but not for women. The prevalence of flexitarians remained stable during the survey period $(15 \cdot 6 \%)$. Over the 13 years, beef intake significantly decreased by $15 \%$ for women and by $9 \%$ for men, which was barely non-significant $(P=0.06)$, while poultry intake significantly increased by 8 and $10 \%$, respectively. Despite those variations, the overall total meat intake remained stable (Table 3). When adjusted for education, occupation and income, there was no longer a significant decrease in poultry intake (online Supplementary Table S1).

\section{Sociodemographic characteristics of different diet groups}

Sociodemographic determinants of dietary choices are presented in Table 4. Compared with omnivores, vegetarians were younger (OR $2.38 ; 95 \%$ CI 1.01, 5.6) and more likely to be women (OR 1.52; $95 \%$ CI 0.98, 2.35), although this result was non-significant. They were also more likely to have a university degree than omnivores (OR 1.59; $95 \%$ CI 1.01, 2.49). Compared with omnivores, pescatarians were more likely to be women (OR 1.81; $95 \%$ CI $1.10,3.00$ ) and less likely to be Swiss (OR $0.59 ; 95 \%$ CI $0.36,0.98)$. Flexitarians were more likely to be female (OR 1.57; $95 \%$ CI 1.41, 1.75), more likely to live alone than omnivores (OR 1.49; $95 \%$ CI 1.33, 1.66) and more likely to be Swiss (OR 1.57; $95 \%$ CI 1.38, 1.77). Conversely, individuals with a low income were more likely to follow a vegetarian (OR 1.83; $95 \%$ CI 1.04, 3.21) or flexitarian (OR 1.31; $95 \%$ CI $1 \cdot 13,1.53)$ diet. Smoking status was not associated with dietary regimen.

\section{Cardiovascular risk factors}

The distribution of cardiovascular risk factor for each dietary regimen is presented in Table 4. Vegetarians were less likely to be overweight (OR $0.55 ; 95 \%$ CI $0.31,0.99$ ), obese (OR 0.27; $95 \%$ CI 0.08, 0.89), hypercholesterolaemic (OR 0.25; $95 \%$ CI $0 \cdot 12,0 \cdot 49$ ) and hypertensive (OR 0.45; $95 \%$ CI 0.23, 0.89) when compared with omnivores. Pescatarians were less likely to be obese (OR 0.18; $95 \%$ CI 0.04, 0.75) and less hypercholesterolaemic (OR 0.53; $95 \%$ CI 0.29, 0.95) when compared with omnivores. Finally, flexitarians were generally less likely to be overweight (OR $0.72 ; 95 \%$ CI $0.63,0.82$ ), obese (OR 0.75; $95 \%$ CI 0.63, 0.90) and hypertensive (OR 0.84; $95 \%$ CI $0.73,0.96)$ than omnivores. Dietary regimen did not influence

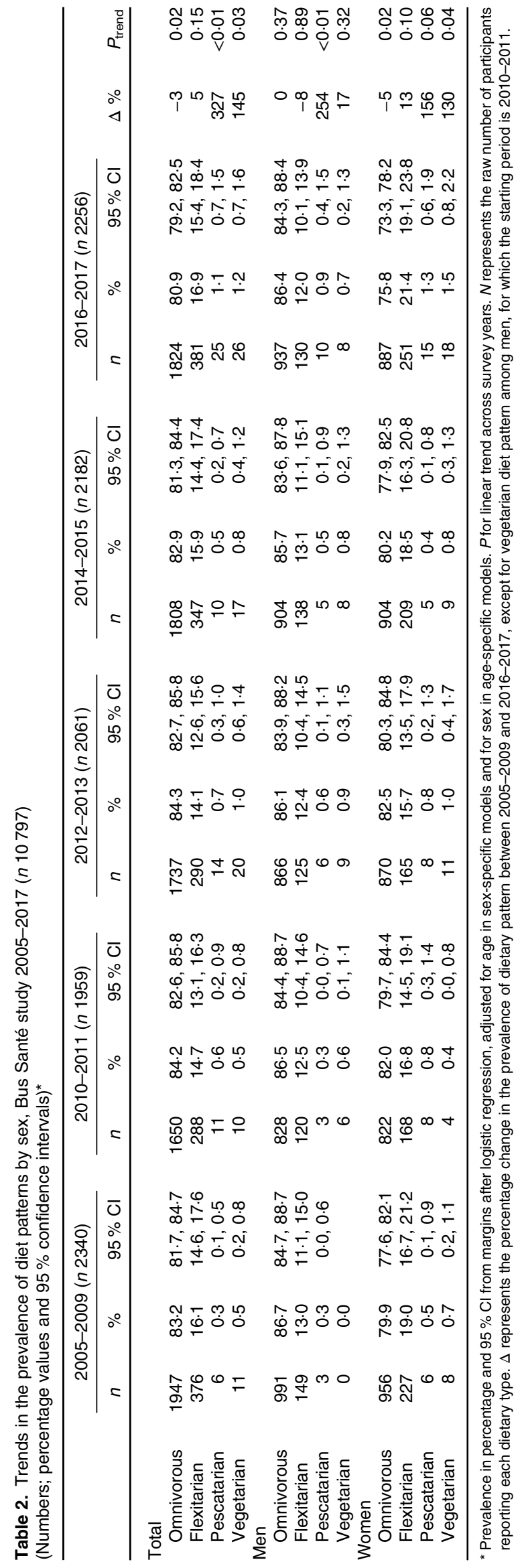


Table 3. Trends in the mean consumption of meat type by sex, Bus Santé study 2005-2017

(Mean values and $95 \%$ confidence intervals)

\begin{tabular}{|c|c|c|c|c|c|c|c|c|c|c|c|c|}
\hline & \multicolumn{2}{|c|}{ 2005-2009 } & \multicolumn{2}{|c|}{ 2010-2011 } & \multicolumn{2}{|c|}{ 2012-2013 } & \multicolumn{2}{|c|}{ 2014-2015 } & \multicolumn{2}{|c|}{ 2016-2017 } & \multirow[b]{2}{*}{$\%$ Change } & \multirow[b]{2}{*}{$P_{\text {trend }}$} \\
\hline & Mean & $95 \% \mathrm{Cl}$ & Mean & $95 \% \mathrm{Cl}$ & Mean & $95 \% \mathrm{Cl}$ & Mean & $95 \% \mathrm{Cl}$ & Mean & $95 \% \mathrm{Cl}$ & & \\
\hline \multicolumn{13}{|l|}{ Men } \\
\hline All meat $(\mathrm{g} / \mathrm{d})$ & 300 & 292,308 & 305 & 297,314 & 304 & 296, 312 & 299 & 291,308 & 295 & 287,303 & -2 & 0.67 \\
\hline Beef $(g / d)$ & 133 & 129,138 & 132 & 127,137 & 131 & 127,136 & 127 & 123,132 & 122 & 117,127 & -9 & 0.06 \\
\hline Processed meat $(\mathrm{g} / \mathrm{d})$ & 35 & 34,37 & 34 & 32,35 & 35 & 34,37 & 35 & 33,36 & 33 & 31,34 & -9 & 0.43 \\
\hline Poultry $(\mathrm{g} / \mathrm{d})$ & 48 & 46,51 & 52 & 50,55 & 51 & 49,54 & 53 & 51,56 & 53 & 50,55 & 8 & 0.04 \\
\hline Fish $(\mathrm{g} / \mathrm{d})$ & 83 & 79,87 & 87 & 83,91 & 86 & 82,90 & 84 & 81,88 & 88 & 84,91 & 5 & 0.44 \\
\hline \multicolumn{13}{|l|}{ Women } \\
\hline All meat $(\mathrm{g} / \mathrm{d})$ & 228 & 220,236 & 233 & 224,241 & 232 & 223,240 & 227 & 219,235 & 223 & 215,231 & -2 & 0.17 \\
\hline Beef $(g / d)$ & 84 & 80,89 & 83 & 78,88 & 82 & 78,87 & 78 & 74,83 & 73 & 68,78 & -15 & $<0.001$ \\
\hline Processed meat $(\mathrm{g} / \mathrm{d})$ & 21 & 20,23 & 19 & 18,21 & 21 & 19,23 & 20 & 19,22 & 19 & 17,20 & -15 & 0.07 \\
\hline Poultry $(\mathrm{g} / \mathrm{d})$ & 43 & 40,45 & 47 & 44,49 & 46 & 43,48 & 48 & 45,50 & 47 & 45,50 & 10 & 0.05 \\
\hline Fish $(\mathrm{g} / \mathrm{d})$ & 79 & 76,83 & 83 & 80,87 & 82 & 78,86 & 81 & 77,84 & 84 & 80,88 & 6 & 0.31 \\
\hline
\end{tabular}

${ }^{*}$ Mean and $95 \% \mathrm{Cl}$ are from margins after linear regression with survey period as predictor, adjusted for age and sex. $P$ for linear trend across survey years.

Table 4. Association of diet patterns with sociodemographic and cardiovascular risk factors, Bus Santé study 2005-2017* $(n 10797)$ (Numbers and percentages; odds ratios and $95 \%$ confidence intervals)

\begin{tabular}{|c|c|c|c|c|c|c|c|c|c|c|c|c|c|c|}
\hline & \multicolumn{2}{|c|}{ Omnivorous } & \multicolumn{2}{|c|}{ Flexitarian } & \multicolumn{2}{|c|}{ Pescatarian } & \multicolumn{2}{|c|}{ Vegetarian } & \multicolumn{2}{|c|}{$\begin{array}{l}\text { Flexitarian } v \text {. } \\
\text { omnivorous }\end{array}$} & \multicolumn{2}{|c|}{$\begin{array}{l}\text { Pescatarian } v \text {. } \\
\text { omnivorous }\end{array}$} & \multicolumn{2}{|c|}{$\begin{array}{l}\text { Vegetarian } v \text {. } \\
\text { omnivorous }\end{array}$} \\
\hline & $n$ & $\%$ & $n$ & $\%$ & $n$ & $\%$ & $n$ & $\%$ & OR & $95 \% \mathrm{Cl}$ & OR & $95 \% \mathrm{Cl}$ & OR & $95 \% \mathrm{Cl}$ \\
\hline $\begin{array}{l}\text { Men } \\
\text { Age categories }\end{array}$ & \multicolumn{8}{|c|}{ Age categories } & 1.00 & Ref. & 1.00 & Ref. & 1.00 & Ref. \\
\hline $18-44$ & 3708 & $41 \cdot 4$ & 670 & $39 \cdot 8$ & 27 & $40 \cdot 9$ & 45 & 53.6 & 0.86 & $0.73,1.01$ & 0.60 & $0.31,1 \cdot 15$ & $2 \cdot 38$ & $1 \cdot 01,5 \cdot 61$ \\
\hline $45-64$ & 3950 & $44 \cdot 1$ & 750 & $44 \cdot 6$ & 25 & 37.9 & 33 & 39.3 & 0.92 & $0.78,1.08$ & 0.58 & $0.30,1.12$ & 1.79 & $0.75,4.27$ \\
\hline $65+$ & 1307 & 14.6 & 262 & $15 \cdot 6$ & 14 & $21 \cdot 2$ & 6 & $7 \cdot 1$ & 1.00 & Ref. & 1.00 & Ref. & 1.00 & Ref. \\
\hline Living alone & 2852 & 33.4 & 680 & $42 \cdot 3$ & 26 & $40 \cdot 0$ & 38 & $45 \cdot 8$ & 1.49 & $1.33,1.66$ & 1.35 & $0.82,2 \cdot 23$ & 1.48 & $0.95,2.29$ \\
\hline Swiss nationality & 5536 & 64.9 & 1203 & 74.9 & 35 & 53.8 & 53 & 63.9 & 1.57 & $1 \cdot 38,1 \cdot 77$ & 0.59 & $0.36,0.98$ & 1.07 & $0.68,1.70$ \\
\hline University degree & 3878 & $46 \cdot 1$ & 773 & $48 \cdot 8$ & 35 & 54.7 & 49 & $60 \cdot 5$ & 1.07 & $0.96,1.20$ & 1.34 & $0.82,2 \cdot 20$ & 1.59 & $1.01,2.49$ \\
\hline Manual occupation & 2263 & $27 \cdot 9$ & 362 & $23 \cdot 7$ & 10 & $16 \cdot 1$ & 17 & $22 \cdot 4$ & 0.85 & $0.74,0.97$ & 0.55 & $0.28,1.09$ & 0.80 & $0.47,1.38$ \\
\hline \multicolumn{15}{|l|}{ Household income } \\
\hline$<5000 \mathrm{CHF} /$ month & 1663 & 21.4 & 374 & $25 \cdot 4$ & 17 & 28.8 & 24 & $32 \cdot 0$ & $1 \cdot 31$ & $1.13,1.53$ & 1.47 & $0 \cdot 76,2 \cdot 83$ & 1.83 & $1.04,3.21$ \\
\hline 5000-9500 CHF/month & 3039 & $39 \cdot 2$ & 582 & 39.5 & 22 & $37 \cdot 3$ & 25 & 33.3 & $1 \cdot 10$ & $0.97,1.26$ & 1.05 & $0.57,1.95$ & 1.01 & $0.58,1.76$ \\
\hline >9500 CHF/month & 3057 & 39.4 & 518 & $35 \cdot 1$ & 20 & 33.9 & 26 & 34.7 & 1.00 & Ref. & 1.00 & Ref. & 1.00 & Ref. \\
\hline Current smoker & 1879 & $22 \cdot 0$ & 365 & $22 \cdot 7$ & 7 & $10 \cdot 8$ & 13 & $15 \cdot 7$ & 1.06 & $0.93,1.21$ & 0.45 & $0.21,1.00$ & 0.59 & $0.33,1.08$ \\
\hline \multicolumn{15}{|l|}{ BMI categories } \\
\hline Overweight & 2945 & 34.5 & 433 & $27 \cdot 4$ & 20 & $31 \cdot 3$ & 16 & $22 \cdot 9$ & 0.72 & $0.63,0.82$ & 0.75 & $0.43,1.31$ & 0.55 & $0.31,0.99$ \\
\hline Obese & 1172 & $13 \cdot 7$ & 183 & 11.6 & 2 & $3 \cdot 1$ & 3 & $4 \cdot 3$ & 0.75 & $0.63,0.90$ & 0.18 & $0.04,0.75$ & 0.27 & $0.08,0.89$ \\
\hline Hypercholesterolaemia & 3289 & $42 \cdot 2$ & 608 & $40 \cdot 5$ & 18 & 29.5 & 10 & $13 \cdot 3$ & 0.95 & $0.84,1.08$ & 0.53 & $0.29,0.95$ & 0.25 & $0.12,0.49$ \\
\hline Hypertension & 2531 & $30 \cdot 4$ & 423 & $26 \cdot 8$ & 16 & $25 \cdot 0$ & 11 & 13.4 & 0.84 & $0.73,0.96$ & 0.70 & $0.38,1.29$ & 0.45 & $0.23,0.89$ \\
\hline Diabetes & 547 & 6.9 & 102 & 6.7 & 2 & $3 \cdot 3$ & 3 & 3.8 & 1.04 & $0.83,1.30$ & 0.44 & $0.11,1.86$ & 0.75 & $0.23,2.42$ \\
\hline
\end{tabular}

CHF, Swiss Francs (1 CHF $=1.01$ USD as of 14 November 2019).

* OR and $95 \% \mathrm{Cl}$ from logistic or multinomial logistic regression models with the diet type as predictor, adjusted for age, sex and survey year. Hypertension was defined as having a previous diagnosis or blood pressure $\geq 140 / 90 \mathrm{mmHg}$. Hypercholesterolaemia was defined as having a previous diagnosis or having total blood cholesterol $>6.5 \mathrm{mmol} / \mathrm{l}$ and $\mathrm{HDL}$ $<1 \mathrm{mmol} / \mathrm{l}$. Diabetes was defined as self-reported diabetes or a fasting plasma glucose level of $\geq 7 \mathrm{mmol} / \mathrm{l}$.

diabetes status. BMI explained part of the association between flexitarians, vegetarians diets and hypertension as well as between pescatarians and hypercholesterolaemia, so that these associations were no longer significant after accounting for BMI (online Supplementary Table S2). Adjustment for education, occupation and income did not change the significance or the magnitude of our results (online Supplementary Table S3).

Association between cardiovascular risk factors on a continuous scale and dietary choices is presented in Table 5 . Vegetarians had a lower BMI $-2.22(95 \%$ CI $-3 \cdot 13,-1 \cdot 31) \mathrm{kg} / \mathrm{m}^{2}$, total cholesterol $-0.44 \quad(95 \%$ CI $-0.67,-0.21) \mathrm{mmol} / \mathrm{l}$ and
LDL $-0.33(95 \%$ CI $-0.52,-0 \cdot 13) \mathrm{mmol} / \mathrm{l}$. Pescatarians had a lower BMI at $-1.7(95 \% \mathrm{CI}-2 \cdot 72,-0 \cdot 68) \mathrm{kg} / \mathrm{m}^{2}$, a lower total cholesterol at $-0.34(95 \% \mathrm{CI}-0.60,-0.08) \mathrm{mmol} / \mathrm{l}$ and LDL $-0.36(95 \% \mathrm{CI}-0.58,-0 \cdot 14) \mathrm{mmol} / \mathrm{l}$. They also had a lower blood pressure with systolic at -4.90 (95\% CI $-8.54,-1 \cdot 27)$ $\mathrm{mmHg}$ and diastolic at -3.31 (95\% CI -5.81, -0.81) $\mathrm{mmHg}$. Flexitarians also had a significant lower BMI at -0.66 (95\% CI $-0.88,-0.44) \mathrm{kg} / \mathrm{m}^{2}$ and a lower blood pressure with systolic at $-0.97(95 \% \mathrm{CI}-1.76,-0.17) \mathrm{mmHg}$ and diastolic at -0.66 (95\% CI $-1.21,-0.11) \mathrm{mmHg}$. Adjustment for education, occupation and income did not change the significance or the magnitude of our results (online Supplementary Table S4). 


\section{Discussion}

In this 13 year cross-sectional population-based study, we showed that the prevalence of vegetarians in the Geneva adult population was low and slightly increased from 0.5 to $1.2 \%$ from 2005-2009 to 2016-2017. An additional 1.1\% of the population was pescatarian in 2016-2017, and flexitarians represented $15.6 \%$ of the studied population and remained stable during the study period. Vegans represented less than $0 \cdot 1 \%$ of the studied population. Compared with omnivores, vegetarians were more likely to be young, have a higher education and a low income; pescatarians were more likely to be women and flexitarians were more likely to be women and had a lower income. Total meat intake remained stable, but both sex reduced their red meat consumption during the studied period with results barely non-significant for men. All dietary regimens excluding/ reducing meat intake showed a more favourable cardiovascular profile compared with omnivorous (i.e. lower BMI, lower total cholesterol and hypertension).

The prevalence of vegetarians observed in previous studies varies greatly. Our study found a mean prevalence in vegetarian diet of $0.8 \%$, which is relatively similar to that reported in a Finnish study ${ }^{(8)}$. The present study was also a cross-sectional examination and used an FFQ to determine the type of diet. The prevalence was slightly lower than that in our study with $0.43 \%$ vegetarians. Others studies have found higher prevalence rates, ranging from 1.7 to $3.9 \%$ in Europe ${ }^{(6,12,14)}$ and in the $\mathrm{USA}^{(13,36)}$. Between-study differences in the prevalence of vegetarians could be due to different methods used to identify the type of diet. Indeed, evidence suggests that when people self-report themselves as vegetarians, higher rates are found than when the study design uses a specific questionnaire like the $\mathrm{FFQ}^{(8)}$. This could be due to a misunderstanding of the definition of vegetarian, which can have a different meaning for each participant and being influenced by cultural norms or health literacy. In addition, it is likely that the population associates vegetarianism with a positive behaviour and wishes to be associated to it, thus producing an overestimation of its true prevalence $^{(8)}$. For instance, the Swiss Vegetarian association estimates the prevalence of vegetarianism in Switzerland in 2017 to be as high as $14 \%$ (3\% vegans, $11 \%$ vegetarians $)^{(37)}$, but these latter estimates are based on a poll that included self-defined vegetarians rather than on a report on actual food intake as in our study. We also observed a positive trend with the prevalence of the vegetarians having increased from $0.5 \%$ at the beginning of the study to $1.2 \%$ at the end. Several studies mention an increase in vegetarianism, but they only cite surveys conducted by vegetarian associations that may tend to overestimate the prevalence of vegetarians ${ }^{(2,4,5)}$. Two independent population-based studies have assessed trends on the prevalence of vegetarian diets, but they presented limitations in their definition of vegetarians ${ }^{(6,36)}$. One of these focused on self-reported vegetarians for health reasons and showed an increase from 1.6 to $1.9 \%$ between 2002 and 2012 in the USA ${ }^{(36)}$. Our study provides new insight based on an independent data source.

In the present study, vegetarians were younger, had a higher level of education and were more likely to be women compared with omnivores, confirming the findings of previous studies ${ }^{(8,9)}$. 
The association between being vegetarian and higher level of education could be explained by the fact that individuals having a higher education level may be more health ${ }^{(38)}$ and/or environment ${ }^{(39)}$ conscious. Alternatively, this association may be due to secular trends in education that imply that young individuals have higher educational levels ${ }^{(17)}$. The latter seems less likely since the analyses were age adjusted. Income was inversely associated with the following vegetarian diet, with participants with a higher income being more likely to be omnivores in our study. The link between income and meat consumption seems to vary considerably depending on the country. For instance, a German study found no association between following a vegetarian diet and income ${ }^{(11)}$, whereas a Canadian and French study found an association between low income and the use of a vegetarian $\operatorname{diet}^{(9,18)}$. In Switzerland, the price of meat ranks among the most expensive in the world ${ }^{(40)}$. For low-income individuals, meat prices could be a reason to reduce meat consumption ${ }^{(9)}$. Meat consumption is highly dependent on cultural habits and social background, which may explain a variation between countries ${ }^{(11)}$.

Our study found that $15.6 \%$ of the population could be defined as flexitarians. To the best of our knowledge, this is the first population-based study to precisely measure with an FFQ the prevalence of this type of diet. Our prevalence of flexitarians seems relatively similar to another study from the Netherlands ${ }^{(12)}$, which reported a prevalence rate of $11-15 \%$ flexitarians between 2009 and 2011, even though in the present study being flexitarian was self-reported ${ }^{(12)}$. Sociodemographic data on flexitarians are lacking in the literature. Flexitarians were more likely to be female and had a lower income than omnivores. However, unlike vegetarians, they did not have higher education and were not younger than omnivores. Interestingly, flexitarians seem to be a distinct population from vegetarians.

Over the 13 years, the overall total meat intake and processed meat remained stable. However, beef intake decreased by $15 \%$ for women and by $9 \%$ for men, and an increase in poultry consumption was observed. This result is interesting as it suggests that the population may be receptive to public health messages encouraging the reduction of red and processed meat intake. Indeed, red and processed meat intake has been positively associated with higher incidence of CVD, type 2 diabetes, certain cancers $^{(3,41,42)}$ and a higher mortality risk ${ }^{(41)}$.

Compared with omnivores, individuals with reduced meat intake generally showed a more favourable profile in terms of cardiovascular risk factors. All dietary profiles had a lower BMI compared with the omnivores (vegetarians of $-2 \cdot 2 \mathrm{~kg} / \mathrm{m}^{2}$, pescatarians of $-1.7 \mathrm{~kg} / \mathrm{m}^{2}$ and flexitarian of $-0.7 \mathrm{~kg} / \mathrm{m}^{2}$ ). Both vegetarians and pescatarians had lower rates of hypercholesterolaemia, and flexitarians had lower values of total cholesterol and LDL. Flexitarians and vegetarians had lower rates of hypertension, and all three diets had lower values of blood pressure compared with omnivores with results barely non-significant for vegetarians, probably due to our small sample size. Adjustment for education, occupation and income did not alter the significance or magnitude of our results. However, as expected, several of the observed associations between diet and cardiovascular risk factors other than BMI seemed to be mediated by BMI, supporting the hypothesis that vegetarian diets may lead to a lower BMI, which could then result in a decrease in cardiovascular risk factors. Overall, our findings confirm the previous results from cross-sectional studies and prospective cohorts ${ }^{(43-45)}$ on the positive link between different vegetarian diets and a positive cardiometabolic profile ${ }^{(17,20,46-50)}$.

It has been proposed that the positive impact on health of vegetarian diets is due to a lower energy density, a lower exposure to harmful components contained in animal food (such as saturated fats, cholesterol, haeme Fe and N-glycolylneuraminic acid) and an increased consumption of protective elements such as fibres and antioxidants ${ }^{(51)}$, along with the positive impact on weight discussed earlier. Although the cross-sectional nature of our study does not allow us to conclude on the causal link between vegetarian diets and cardiovascular risk factors, our results are in line with those from important longitudinal studies and several randomised controlled trials reporting the beneficial effects of a vegetarian diet, with less evidence for flexitarians ${ }^{(24,43,44,46,48,52,53)}$. Finally, we cannot exclude that these described positive effects may be due to unmeasured confounding factors. For example, these beneficial effects may be related to the generally advantaged socio-behavioural profile of vegetarians. In our study, this bias seems to be limited by the fact that our population of vegetarians had a lower income than omnivores, with low income generally being a cardiovascular risk factor $^{(54)}$.

Some limitations to our study need to be acknowledged. The sample of vegetarians was low, which potentially leads to power issues. Moreover, the study design did not allow to account for how long participants had been following a specific diet. Since an individual's meat consumption can fluctuate from month to month, it is possible that an FFQ conducted over a period of only 4 weeks could lead to a misclassification bias and thus mitigate the overall results. In addition to this, although the Bus Santé study aims to be representative of the Geneva population, recruitment bias cannot be excluded. This could lead to an over- or under-selection of participants with different profiles from the population. However, the prevalence of cardiovascular risk factors found in our study appears to be similar to that found in the general population of Geneva ${ }^{(55)}$.

Our study has several strengths. Unlike other studies where the data came from self-reported online ${ }^{(9)}$ or mailed $^{(19)}$ questionnaires, in our study, the questionnaires, anthropometric measurements and biological data came from standardised measurements performed by trained personnel. Unlike many previous studies ${ }^{(8,10,11)}$, vegetarians were identified through an FFQ rather than a subjective question on the type of diet that participants were following. Evidence has shown that up to $80 \%$ of self-reported vegetarians were in fact omnivores ${ }^{(8)}$. Finally, our results are obtained from a cross-sectional study that was government funded with a recruitment in the general population, independent from parties with a potential conflict of interests such as the industry or vegetarian associations. All these strengths should result in more reliable and objective data. 


\section{Conclusion}

In our cross-sectional population study spanning over 13 years, the proportion of vegetarians and pescatarians in the population increased, whereas that of flexitarians remained stable at a remarkably high rate. Vegetarians were younger, had a lower income, higher education and were more likely to be women. Like vegetarians, flexitarians were more prone to be female and had a lower income but contrary to vegetarians they did not have higher education and were not younger than omnivores, suggesting that this population represents a distinct pattern among the different vegetarians diet. Vegetarians, pescatarians and flexitarians had a lower prevalence of cardiovascular risks factors such as high BMI, hypertension or hypercholesterolaemia. Our results confirm previous reports from longitudinal and randomised controlled trials and reiterate that fact that promoting a reduction of meat consumption would not only benefit the planet but also population health.

\section{Acknowledgements}

This work was funded with grant from a donor of the Private Foundation of the Geneva University Hospitals. The Private Foundation of the Geneva University Hospitals had no role in the design, analysis or writing of this article.

S. S., H. W. and C. L. designed research; S. S. and C. d. M. performed statistical analysis; S. S., H. W., C. L., J.-L. R. and I. G. wrote the paper; S. S. had primary responsibility for final content. All authors read and approved the final manuscript.

The authors declare that they have no conflicts of interest.

\section{Supplementary material}

For supplementary material referred to in this article, please visit https://doi.org/10.1017/S0007114520001762

\section{References}

1. Forestell CA (2018) Flexitarian diet and weight control: healthy or risky eating behavior? Front Nutr 5, 59.

2. Ruby MB (2012) Vegetarianism. A blossoming field of study. Appetite 58, 141-150.

3. McEvoy CT, Temple N \& Woodside JV (2012) Vegetarian diets, low-meat diets and health: a review. Public Health Nutr 15, 2287-2294.

4. Bieri A, von Siebenthal C \& Köhler H (2018) Alimentation végétarienne et végane chez les enfants et adolescents (Vegetarian and vegan diets in children and teenagers). Swiss Med Forum 18, 393-398.

5. Leitzmann C (2014) Vegetarian nutrition: past, present, future. Am J Clin Nutr 100, 496-502.

6. ANSES (2017) Étude individuelle nationale des consommations alimentaires 3 (INCA 3). https://www.anses.fr/fr/system/files/ NUT2014SA0234Ra.pdf (accessed June 2019).

7. Leung B, Lauche R, Leach M, et al. (2018) Special diets in modern America: analysis of the 2012 National Health Interview Survey data. Nutr Health 24, 11-18.

8. Vinnari M, Montonen J, Härkänen T, et al. (2009) Identifying vegetarians and their food consumption according to self-identification and operationalized definition in Finland. Public Health Nutr 12, 481-488.

9. Allès B, Baudry J, Méjean C, et al. (2017) Comparison of sociodemographic and nutritional characteristics between self-reported vegetarians, vegans, and meat-eaters from the nutrinet-santé study. Nutrients $\mathbf{9}, 1023$.

10. Ponzio E, Mazzarini G, Gasperi G, et al. (2015) The vegetarian habit in Italy: prevalence and characteristics of consumers. Ecol Food Nutr 54, 370-379.

11. Pfeiler TM \& Egloff B (2018) Examining the 'Veggie' personality: results from a representative German sample. Appetite 120, 246-255.

12. Dagevos H (2014) Flexibility in the frequency of meat consumption - empirical evidence from The Netherlands. EuroChoices 13, 40-45.

13. Kim H, Rotundo L, Song D, et al. (2017) The prevalence and characteristics of vegetarian in the United States: a population-based study. Gastroenterology 152, S1016.

14. Newby PK, Tucker KL \& Wolk A (2005) Risk of overweight and obesity among semivegetarian, lactovegetarian, and vegan women. Am J Clin Nutr 81, 1267-1274.

15. Melina V, Craig W \& Levin S (2016) Position of the Academy of Nutrition and Dietetics: vegetarian diets. J Acad Nutr Diet 116, 1970-1980.

16. Derbyshire EJ (2017) Flexitarian diets and health: a review of the evidence-based literature. Front Nutr 3, 55.

17. Knox KH, Allen NE, Spencer EA, et al. (2003) EPIC-Oxford: lifestyle characteristics and nutrient intakes in a cohort of 33883 meat-eaters and 31546 non meat-eaters in the UK. Public Health Nutr 6, 259-268.

18. Bedford JL \& Barr SI (2005) Diets and selected lifestyle practices of self-defined adult vegetarians from a population-based sample suggest they are more 'health conscious'. Int J Behav Nutr Phys Act 2, 4

19. Tonstad S, Butler T, Yan R, et al. (2009) Type of vegetarian diet, body weight, and prevalence of type 2 diabetes. Diabetes Care 32, 791-796.

20. Tonstad S, Stewart K, Oda K, et al. (2013) Vegetarian diets and incidence of diabetes in the Adventist Health Study-2. Nutr Metab Cardiovasc Dis 23, 292-299.

21. Dinu M, Abbate R, Gensini GF, et al. (2017) Vegetarian, vegan diets and multiple health outcomes: a systematic review with meta-analysis of observational studies. Crit Rev Food Sci Nutr 57, 3640-3649.

22. Nakamoto K, Watanabe S, Kudo H, et al. (2008) Nutritional characteristics of middle-aged Japanese vegetarians. I Atheroscler Thromb 15, 122-129.

23. Valachovičová M, Príbojová J, Urbánek V, et al. (2017) Selected cardiovascular risk markers in vegetarians and subjects of general population. Cent Eur J Public Health 25, 299-302.

24. Chiu YF, Hsu CC, Chiu THT, et al. (2015) Cross-sectional and longitudinal comparisons of metabolic profiles between vegetarian and non-vegetarian subjects: a matched cohort study. BrJ Nutr 114, 1313-1320.

25. Yang SY, Li XJ, Zhang W, et al. (2012) Chinese lacto-vegetarian diet exerts favorable effects on metabolic parameters, intimamedia thickness, and cardiovascular risks in healthy men. Nutr Clin Pract 27, 392-398.

26. Derbyshire EJ (2016) Flexitarian diets and health: a review of the evidence-based literature. Front Nutr 3, 55.

27. Kim H, Caulfield LE, Garcia-Larsen V, et al. (2019) Plant-based diets are associated with a lower risk of incident cardiovascular disease, cardiovascular disease mortality, and all-cause mortality in a general population of middle-aged adults. J Am Heart Assoc 8, e012865. 
28. Mihrshahi S, Ding D, Gale J, et al. (2017) Vegetarian diet and allcause mortality: evidence from a large population-based Australian cohort - the 45 and Up Study. Prev Med (Baltim) 97, 1-7.

29. Guessous I, Luthi J-C, Bowling CB, et al. (2014) Prevalence of frailty indicators and association with socioeconomic status in middle-aged and older adults in a Swiss region with universal health insurance coverage: a population-based cross-sectional study. J Aging Res 2014, 198603.

30. Guessous I, Joost S, Jeannot E, et al. (2014) A comparison of the spatial dependence of body mass index among adults and children in a Swiss general population. Nutr Diabetes 4, e111.

31. Bernstein M, Morabia A, Costanza MC, et al. (1994) Equilibre nutritionnel de l'alimentation de la population adulte résidant à Genève (Nutritional balance of the diet of the adult population living in Geneva). Sozial- und Präventivmedizin SPM 39 333-344.

32. De Abreu D, Guessous I, Gaspoz JM, et al. (2014) Compliance with the Swiss society for nutrition's dietary recommendations in the population of Geneva, Switzerland: a 10-year trend study (1999-2009). J Acad Nutr Diet 114, 774-780.

33. Morabia A, Bernstein M, Héritier S, et al. (1997) Communitybased surveillance of cardiovascular risk factors in Geneva: methods, resulting distributions, and comparisons with other populations. Prev Med (Baltim) 26, 311-319.

34. Morabia A, Bernstein M, Kumanyika S, et al. (1994) Développement et validation d'un questionnaire alimentaire semi-quantitatif à partir d'une enquête de population (Development and validation of a semi-quantitative food questionnaire based on a population survey). Soz Präventivmed 39, 345-369.

35. Clarys P, Deliens T, Huybrechts I, et al. (2014) Comparison of nutritional quality of the vegan, vegetarian, semi-vegetarian, pesco-vegetarian and omnivorous diet. Nutrients 6, 1318-1332.

36. Cramer H, Kessler CS, Sundberg T, et al. (2017) Characteristics of Americans choosing vegetarian and vegan diets for health reasons. J Nutr Educ Behav 49, 561-567.e1.

37. Swissveg (2019) Veggie survey 2017. https://www.swissveg. ch/veggie_survey?language=en (accessed June 2019).

38. LiJ \& Powdthavee N (2015) Does more education lead to better health habits? Evidence from the school reforms in Australia. Soc Sci Med 127, 83-91.

39. Duroy QM (2005) The determinants of environmental awareness and behavior. Rensselaer Polytechnic Institute, Working Papers in Economics.

40. Caterwings (2019) Worldwide Meat Price Index - USD, 1-5. https://www.caterwings.co.uk/caterers/meat-price-index-usd/ (accessed August 2019).
41. Zheng Y, Li Y, Satija A, et al. (2019) Association of changes in red meat consumption with total and cause specific mortality among US women and men: two prospective cohort studies. BMJ 365, 12110.

42. Micha R, Wallace SK \& Mozaffarian D (2010) Red and processed meat consumption and risk of incident coronary heart disease, stroke, and diabetes mellitus: a systematic review and metaanalysis. Circulation 121, 2271-2283.

43. Yokoyama Y, Levin SM \& Barnard ND (2017) Association between plant-based diets and plasma lipids: a systematic review and meta-analysis. Nutr Rev 75, 683-698.

44. Yokoyama Y, Nishimura K, Barnard ND, et al. (2014) Vegetarian diets and blood pressure ameta-analysis. JAMA Intern Med 174, 577-587.

45. Picasso MC, Lo-Tayraco JA, Ramos-Villanueva JM, et al. (2019) Effect of vegetarian diets on the presentation of metabolic syndrome or its components: a systematic review and metaanalysis. Clin Nutr 38, 1117-1132.

46. Spencer EA, Appleby PN, Davey GK, et al. (2003) Diet and body mass index in 38000 EPIC-Oxford meat-eaters, fisheaters, vegetarians and vegans. Int J Obes 27, 728-734.

47. Crowe FL, Appleby PN, Travis RC, et al. (2012) Risk of ischaemic heart disease among British vegetarians and nonvegetarians: results from the EPIC-Oxford cohort study. Proc Nutr Soc 71, 597-603.

48. Papier K, Appleby PN, Fensom GK, et al. (2019) Vegetarian diets and risk of hospitalisation or death with diabetes in British adults: results from the EPIC-Oxford study. Nutr Diabetes 9, 7 .

49. Orlich MJ, Singh PM, Sabaté J, et al. (2013) Vegetarian dietary patterns and mortality in Adventist Health Study 2.JAMA Intern Med 173, 1230-1238.

50. Tonstad S, Butler T, Yan R, et al. (2009) Type of vegetarian diet, body weight, and prevalence of type 2 diabetes. Diabetes Care 32, 791-796.

51. Kahleova H, Levin S \& Barnard ND (2018) Vegetarian dietary patterns and cardiovascular disease. Prog Cardiovasc Dis 61, 54-61.

52. Wang F \& Li D (2015) Effects of vegetarian diets on blood lipids : a systematic review and meta-analysis of randomized controlled trials. J Am Heart Assoc 4, e002408.

53. Margetts BM, Armstrong BK, Beilin LJ, et al. (1986) Vegetarian diet in mild hypertension: a randomised controlled trial. Br Med $J$ (Clin Res Ed) 293, 1468-1471.

54. Kreatsoulas C \& Anand SS (2010) The impact of social determinants on cardiovascular disease. Can J Cardiol 26, 8C-13C.

55. Federal Office of Statistics (2017) Geneva Health Statistics. https://www.ge.ch/statistique/ofs/sante2017/files/fr/02b.xml (accessed July 2019). 